\title{
Carbon paste electrode incorporating multi-walled carbon nanotube/ferrocene as a sensor for the electroanalytical determination of $N$-acetyl- $L$-cysteine in the presence of tryptophan
}

\author{
JAHAN BAKHSH RAOOF ${ }^{\mathrm{a}, *}$, FERESHTEH CHEKIN ${ }^{\mathrm{b}}$, REZA OJANI ${ }^{\mathrm{a}}$ \\ and SAEIDEH BARARI ${ }^{\mathrm{a}}$ \\ ${ }^{a}$ Electroanalytical Chemistry Research Laboratory, Department of Analytical Chemistry, \\ Faculty of Chemistry, University of Mazandaran, Babolsar, Iran \\ ${ }^{b}$ Department of Chemistry, Islamic Azad University, Ayatollah Amoli Branch, Amol, Iran \\ e-mail: j.raoof@umz.ac.ir
}

MS received 13 February 2012; revised 25 June 2012; accepted 10 August 2012

\begin{abstract}
The preparation and electrochemical performance of the carbon nanotube paste electrode modified with ferrocene (FCMCNPE) was investigated for electrocatalytic behaviour toward oxidation of $N$-acetyl$L$-cysteine (NAC) in the presence of tryptophan (Trp) using cyclic voltammetry (CV) and differential pulse voltammetry (DPV). The results showed an efficient electrocatalytic activity of FCMCNPE toward oxidation of NAC and Trp, as the electrooxidation of NAC and Trp together gave two well-defined anodic peaks, revealing the applicability of this modified electrode for simultaneous voltammetric detection of mentioned compounds in the same solution. The values of catalytic rate constant $(k)$ and the apparent diffusion coefficient $\left(D_{\text {app }}\right)$ were also calculated using chronoamperometry. The DPV method was applied as a sensitive method for the quantitative detection of trace amounts of NAC and Trp. A linear dynamic range from 1.0 to $18.0 \mu \mathrm{M}$ for NAC and 2.0 to $150.0 \mu \mathrm{M}$ for Trp was obtained using DPV method in $\mathrm{pH} 7.00$ buffered solution and the detection limit $(3 \sigma)$ was determined as $0.49 \mu \mathrm{M}$ and $0.54 \mu \mathrm{M}$ for NAC and Trp, respectively. The proposed method was also applied for analysis of NAC tablet, investigating the applicability of the proposed voltammetric method for determination of NAC in real sample.
\end{abstract}

Keywords. $\quad N$-acetyl- $L$-cysteine; tryptophan; ferrocene; electrocatalysis; carbon nanotube paste electrode.

\section{Introduction}

$N$-acetyl- $L$-cysteine (NAC), as an acetyl derivative and also one of the homologs of $L$-cysteine, is very important amino acid owing to its crucial roles in biological systems. ${ }^{1}$ For example, it could be used as a prospective radiation protector, antitoxin, antioxidant and free radical and reactive oxygen species scavenger. ${ }^{2}$ NAC has been efficient in the treatment of Sjogren's syndrome, influenza, smoking cessation, myoclonus epilepsy and hepatitis $\mathrm{C},{ }^{3}$ cancer, ${ }^{4}$ human immunodeficiency virus (HIV) infection, ${ }^{5}$ cardiovascular and respiratory diseases, ${ }^{6}$ acetaminophen toxicity, ${ }^{7}$ neurodegenerative disorder ${ }^{8}$ and the other diseases characterized by free radicals production and oxidative damage. Numerous chemical and instrumental techniques, such as chromatography, ${ }^{9}$ spectrophotometry ${ }^{10}$ and fluorimetry ${ }^{11}$ were employed for the determination of this compound. However, these methods suffer

*For correspondence from some disadvantages such as high cost, long analysis time and requirement for sample pre-treatment that makes them unsuitable for routine analysis. Compared to these options, electro-analysis is more promising approach, due to simplicity, low expense and high sensitivity. ${ }^{12}$ However, NAC oxidation at bare electrodes requires high overpotential and the corresponding voltammetric signals on the surface of bare electrodes are usually weak that makes its detection unsatisfactory. Tryptophan (2-amino-3-(1H-indol3 -yl)-propionic acid), Trp is essential amino acid for humans and a precursor for serotonin (a neurotransmitter), melatonin (a neurohormone) and niacin. This compound is sometimes added to dietary, food products, pharmaceutical formulas due to the scarcely presence in vegetables. ${ }^{13}$ Tryptophan is known to undergo electrochemical oxidation and several polymeric/oligomeric products can be formed on the electrode surface. ${ }^{14}$

The electrochemical oxidation of NAC and Trp at bare electrode results highly overlapped current responses which make their discrimination very 
difficult and leads to poor selectivity and reproducibility of the electrode. To our knowledge, several electrochemical methods based on the chemical modification of traditional electrode materials, have been developed for determination of NAC (e.g., carbon paste electrode modified with copper(II) hexacyanoferrate(III), ${ }^{15}$ palladized aluminum electrode covered by prussian blue film ${ }^{16}$ and nanocomposite containing naphthoquinone and nanogold modified glassy carbon electrode) ${ }^{17}$ and $\operatorname{Trp}$ (e.g., nano- $\mathrm{TiO}_{2} /$ ferrocene carboxylic acid modified carbon paste electrode ${ }^{18}$ and carbon nanotube paste electrode modified with cobalt salophen), ${ }^{19}$ individually.

Ferrocene and its derivatives are the coordination compounds known as useful and suitable electrode modifier for their excellent electrocatalytic properties toward the detection of many important drugs and bio-subatrates. ${ }^{20-22}$

Application of carbon nanotubes (CNTs) as an important nano-structured material has also recently attracted a lot of attention in electrochemical researches for the determination of biomolecules due to their exceptional properties, such as strong electrocatalytic effect, fast electron transfer rate, high conductance, biocompatibility, high stability and high surface area. ${ }^{23-31}$

The integration of redox mediators and CNTs in the matrix of the modified electrodes is considered as a new and interesting area in the development of electrochemical sensors. ${ }^{32-36}$ In the present work, we describe the preparation of a carbon nanotube paste electrode modified with ferrocene as a redox electron transfer mediator for improvement in the kinetic of the electrode process and sensitivity of the voltammetric measurements. We investigated the suitability of this modified electrode (FCMCNPE) for electrocatalysis of NAC in the presence of Trp. A complete resolution between DPV peaks of NAC and Trp (about $450 \mathrm{mV}$ separation in peak potentials) provides a suitable and effective method for simultaneous determination of these two compounds at the surface of FCMCNPE.

\section{Experimental}

\subsection{Materials and apparatus}

The solvent used for the electrochemical studies was twice distilled water. Phosphate buffered solutions (PBS) 0.1 M for different $\mathrm{pH}$ values were prepared from orthophosphoric acid $\left(\mathrm{H}_{3} \mathrm{PO}_{4}\right)$ and its salts $\left(\mathrm{NaH}_{2} \mathrm{PO}_{4}\right.$, $\mathrm{Na}_{2} \mathrm{HPO}_{4}$ and $\mathrm{Na}_{3} \mathrm{PO}_{4}$ ). High viscosity paraffin (density $=0.88 \mathrm{~g} \mathrm{~cm}^{-3}$ ) from Fluka was used as the pasting liquid for the carbon paste electrode. Graphite powder (particle diameter $=0.1 \mathrm{~mm}$ ) from Merck and multiwalled carbon nanotube (with purity OF $>95 \%$, diameter $54 \mathrm{~nm}$, length $1-10 \mu \mathrm{m}$, number of walls $3-15$, from Nanostartech. Co., Tehran, Iran) were used as the working electrode (WE) substrates. Ferrocene (FC), NAC and Trp were from Fluka. Acetylcysteine tablets (labelled value $600 \mathrm{mg}$, from Zambon Co., imported by Darman Yab Darou Co.) were purchased from a local drugstore. NAC and Trp solutions were prepared daily. All other chemicals used were of analytical reagent grade.

The electrochemical measurements were carried out using a potentiostat/galvanostat (SAMA 500; Electroanalysis System, Iran) connected to Pentium IV personal computer. The electrochemical experiments were performed at room temperature with a three electrode assembly including a carbon paste (unmodified or modified), platinum disk and $\mathrm{Ag}|\mathrm{AgCl}| \mathrm{KCl}(3 \mathrm{M})$ (from Azar Electrode Co., Iran) as a working electrode, auxiliary electrode and reference electrode, respectively. Also, a digital pH-meter (model 780, from Metrohm Co., Switzerland) was used to read the $\mathrm{pH}$ of the buffered solutions.

\subsection{Preparation of working electrode}

FCMCNPE was made by dissolving the mixture of $1 \%(\mathrm{w} / \mathrm{w})$ ferrocene, $10 \%(\mathrm{w} / \mathrm{w})$ multi-walled carbon nanotube and $89 \%(\mathrm{w} / \mathrm{w})$ graphite powder in diethyl ether in a mortar. The mixture was stirred until the solvent was evaporated, completely. Then, the resulting composite and paraffin were blended by handmixing until a uniform paste was obtained. A portion of this paste was packed into the bottom of a glass tube (3.4 $\mathrm{mm}$ i.d.). Electrical contact was made by forcing a copper wire down into the tube. When necessary, a new surface was obtained by pushing out an excess of paste and polishing it on weighing paper. The ferrocene modified carbon paste electrode (FCMCPE) and carbon nanotube-paste electrode (CNPE) was prepared in the same way but without adding CNT and FC, respectively.

\subsection{Preparation of real sample}

For analysis of the NAC tablet, the tablet sample was finely powdered and homogenized. A weighted amount of this powdered sample was dissolved in $0.1 \mathrm{M}$ phosphate buffered solution ( $\mathrm{pH}$ 7.00). Then, a known amount of this aqueous sample of the drug was diluted to the volume of $10 \mathrm{~mL}$ and placed in a voltammetric cell. The standard addition method $(n=3)$ was 
employed for measuring NAC concentration in the sample.

\section{Results and discussion}

\subsection{Electrochemical behaviour of FCMCNPE and FCMCPE in the absence and presence of NAC}

We have previously shown that a carbon paste electrode spiked with ferrocene is constructed by the incorporation of ferrocene in a graphite powder/paraffin oil matrix. ${ }^{37}$ The cyclic voltammograms of FCMCPE and FCMCNPE (figure 1c and d) exhibit one anodic and cathodic peak corresponding with redox system $\mathrm{FC} / \mathrm{FC}^{+}$, which show a quasi-reversible behaviour in an aqueous medium. The cyclic voltammograms of bare CPE and CNPE in pure supporting electrolyte show no anodic or cathodic peaks (figure $1 \mathrm{a}$ and $\mathrm{b}$ ). Also, the comparison of FCMCNPE and CNPE with FCM$\mathrm{CPE}$ and $\mathrm{CPE}$ electrochemical behaviour, respectively shows the presence of CNT with many specific advantages such as large surface area and high conductivity, can provide a suitable interface to promote the electron transfer reaction. Thus, the electrochemical response of $\mathrm{FC}$ will be greatly enhanced on FCMCNPE.

The kinetic parameters $\alpha$ and $k$ for ferrocene were estimated using the model of Laviron: ${ }^{38}$

$$
\begin{aligned}
\log (k)= & \alpha \log (1-\alpha)+(1-\alpha) \log \alpha \\
& -\log (R T / n F v) \\
& -\alpha(1-\alpha) \log \left(n F \Delta E_{p} / 2.3 R T\right),
\end{aligned}
$$

where $\alpha$ is the transfer coefficient, $k$ is the heterogeneous electron-transfer rate constant, $\Delta E_{p}$ is the peak-to-peak separation, $n$ is the number of electrons transferred in the rate-determining reaction, $R$ is the gas

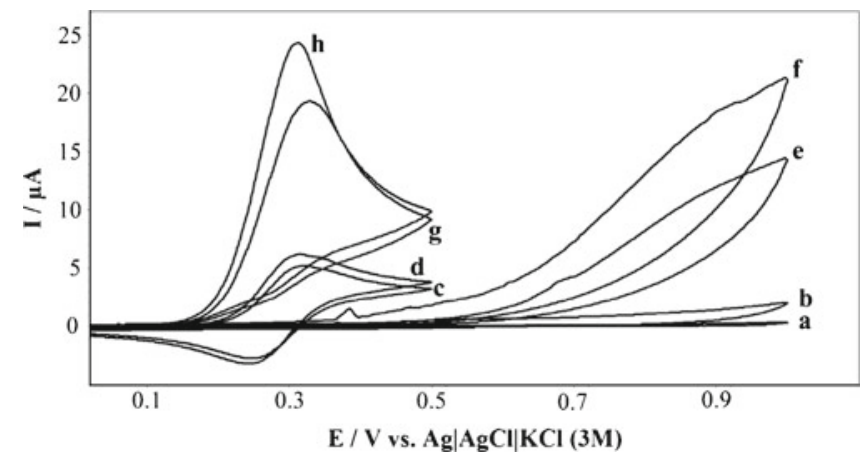

Figure 1. Cyclic voltammograms of (a) CPE, (b) CNPE, (c) FCMCPE and (d) FCMCNPE in the absence of NAC and (e) as (a), (f) as (b), (g) as (c) and (h) as (d) in the presence of $1.0 \mathrm{mM}$ NAC in $0.1 \mathrm{M}$ PBS ( $\mathrm{pH} 7.00)$ at scan rate of potential $20 \mathrm{mV} \mathrm{s}^{-1}$. constant, $T$ is the absolute temperature and $v$ is the scan rate. A graph of $\Delta E_{p}$ versus the logarithm of the scan rate yielded a straight line. Based on this, the values of $\alpha=0.64$ and $k=3.47 \mathrm{~s}^{-1}$ were calculated.

In order to study the electrocatalytic efficiency of the modified electrodes toward NAC oxidation, cyclic voltammetric responses of $1.0 \mathrm{mM}$ NAC were recorded in $0.1 \mathrm{M}$ phosphate buffered solution with $\mathrm{pH} 7.00$ on the surface of modified and unmodified electrodes. As shown in figure 1, NAC oxidation occurs irreversibly with a broad peak at potential of about $850 \mathrm{mV}$ at $\mathrm{CPE}$ (curve e) and CNPE (curve f), while at FCMCPE (curve g) and FCMCNPE (curve h), appears at potential of about $320 \mathrm{mV}$. These results confirm that the oxidation peak potential of NAC at FCMCPE and FCMCNPE is shifted about $530 \mathrm{mV}$ toward less positive potential compared with that at bare CPE and CNPE. In the presence of NAC, the anodic peak current was increased greatly for FCMCPE and FCMCNPE, while the corresponding cathodic peak was disappeared on the reverse scan. All of these observations clearly show efficient electrocatalytic activity of FCMCNPE for lowering the anodic overpotential and improvement in the sensitivity of the voltammetric measurements.

\subsection{Electrochemical behaviour of NAC in the presence of Trp at FCMCNPE}

In order to study the simultaneous determination of NAC and Trp at the surface of FCMCNPE, cyclic voltammograms of FCMCNPE and CNPE in the buffer solutions containing $0.1 \mathrm{mM}$ Trp and $0.5 \mathrm{mM}$ NAC were recorded. As can be seen in figure 2 (curves a and $b$ ), the oxidation peak of NAC overlapped with that of Trp at CNPE, so electrochemical determination of them in their mixture solution is not possible. FC in the matrix of FCMCNPE can act as an efficient catalyst for lowering the anodic overpotential of NAC without any change in the peak potential of Trp (curve c). This effect makes a desirable separation between the oxidation peaks of NAC and Trp.

\subsection{The influence of $\mathrm{pH}$ on the electrochemical properties of $N A C$}

In order to optimize the electrochemical response of FCMCNPE to NAC oxidation, we investigated the effect of solution $\mathrm{pH}$ on the electrochemical behaviour of this compound in $1.0 \mathrm{mM}$ solutions of NAC over a $\mathrm{pH}$ range between 4.0 and 9.0 at the surface of modified electrode using cyclic voltammetry. The results show that in the electrocatalytic oxidation process of NAC, 


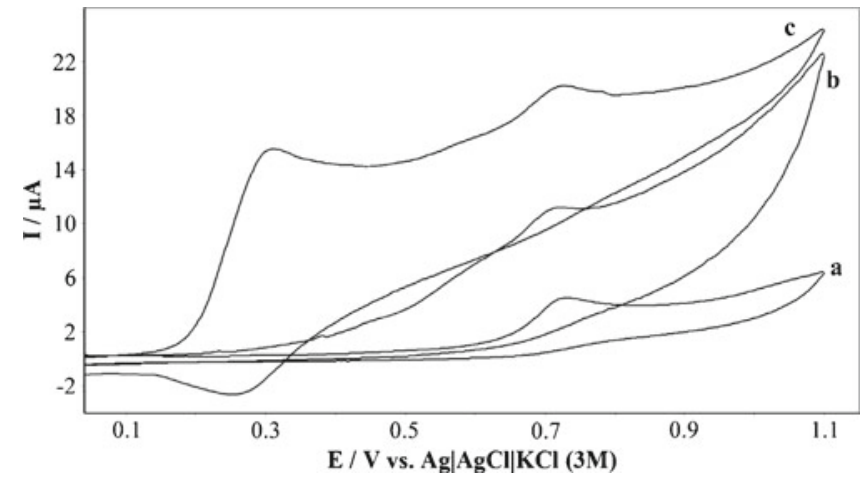

Figure 2. Cyclic voltammograms of (a) $0.1 \mathrm{mM}$ Trp and (b) $0.1 \mathrm{mM}$ Trp + 0.5 mM NAC in 0.1 M PBS (pH 7.00) at CNPE and (c) as (b) at FCMCNPE at scan rate of potential $20 \mathrm{mV} \mathrm{s}^{-1}$.

the anodic peak current reaches to a maximum value in $\mathrm{pH} 7.00$, indicating the maximum electrocatalytic efficiency of FCMCNPE for NAC electro-oxidation in this $\mathrm{pH}$ (not shown). Therefore, $\mathrm{pH} 7.00$ was taken as the optimum $\mathrm{pH}$ for the determination of this compound at FCMCNPE.

\subsection{Effect of potential scan rate}

The effect of the potential scan rate $(v)$ on the electrocatalytic property of FCMCNPE toward electrooxidation of $1.0 \mathrm{mM}$ NAC was studied by cyclic voltammetry (figure 3a). As can be seen in figure $3 b$, over the range of $v=10-300 \mathrm{mVs}^{-1}$, the anodic peak current $\left(\mathrm{I}_{\mathrm{pa}}\right)$ increases linearly with the square root of the potential scan rate, indicating that the process is diffusioncontrolled. In addition, with increasing the potential scan rate, the catalytic oxidation peak potential was shifted toward more positive potentials, suggesting a kinetic limitation in the reaction between the redox site of ferrocene in the FCMCNPE and NAC at higher scan rates.

In order to obtain information on the ratedetermining step, a Tafel plot was drawn by using the rising part of the cyclic voltammograms of FCMCNPE in $0.1 \mathrm{M}$ PBS containing $1.0 \mathrm{mM} \mathrm{NAC}$ at $\nu=20 \mathrm{mVs}^{-1}$ (figure $3 \mathrm{c}$ ). The resulted slope indicates a transfer coefficient $(\alpha)$ equal to 0.71 for one electron transfer process, which is rate-determining step. This value of $\alpha$ is in accordance with that reported data. ${ }^{39}$

\subsection{Chronoamperometry studies}

Double potential step chronoamperometry method was also employed for the investigation of electrode processes. Therefore, we studied the chronoamperometric behaviour of the modified electrode in $0.1 \mathrm{M}$ phosphate buffered solution $(\mathrm{pH} 7.00)$ in the absence and presence of various concentrations of NAC by setting the
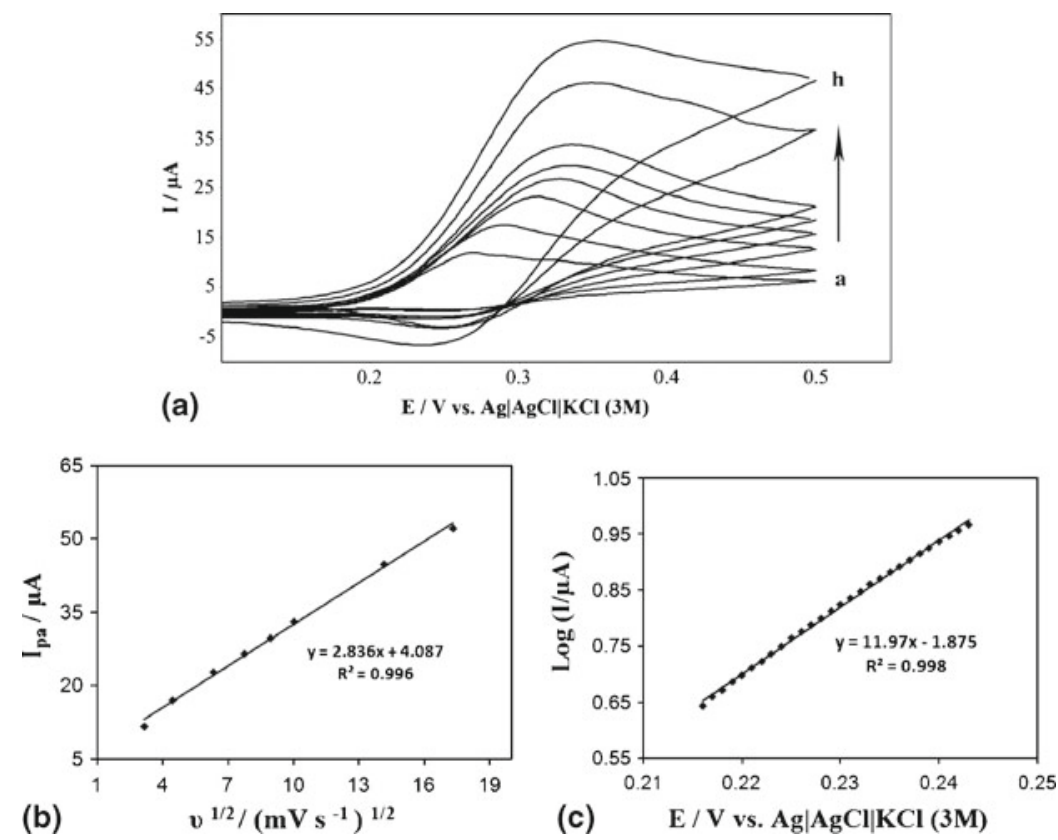

Figure 3. (a) Cyclic voltammograms of FCMCNPE in $0.1 \mathrm{M}$ PBS (pH 7.00) containing $1.0 \mathrm{mM}$ NAC at various potential scan rates (a) 10 , (b) 20, (c) 40, (d) 60, (e) 80, (f) 100, (g) 200 and (h) $300 \mathrm{mVs}^{-1}$. (b) Plot of anodic peak current vs. $v^{1 / 2}$. (c) Tafel plot for FCMCNPE in the presence of 1.0 mM NAC in 0.1 M PBS (pH 7.00) at potential scan rate of $20 \mathrm{mV} \mathrm{s}^{-1}$. 

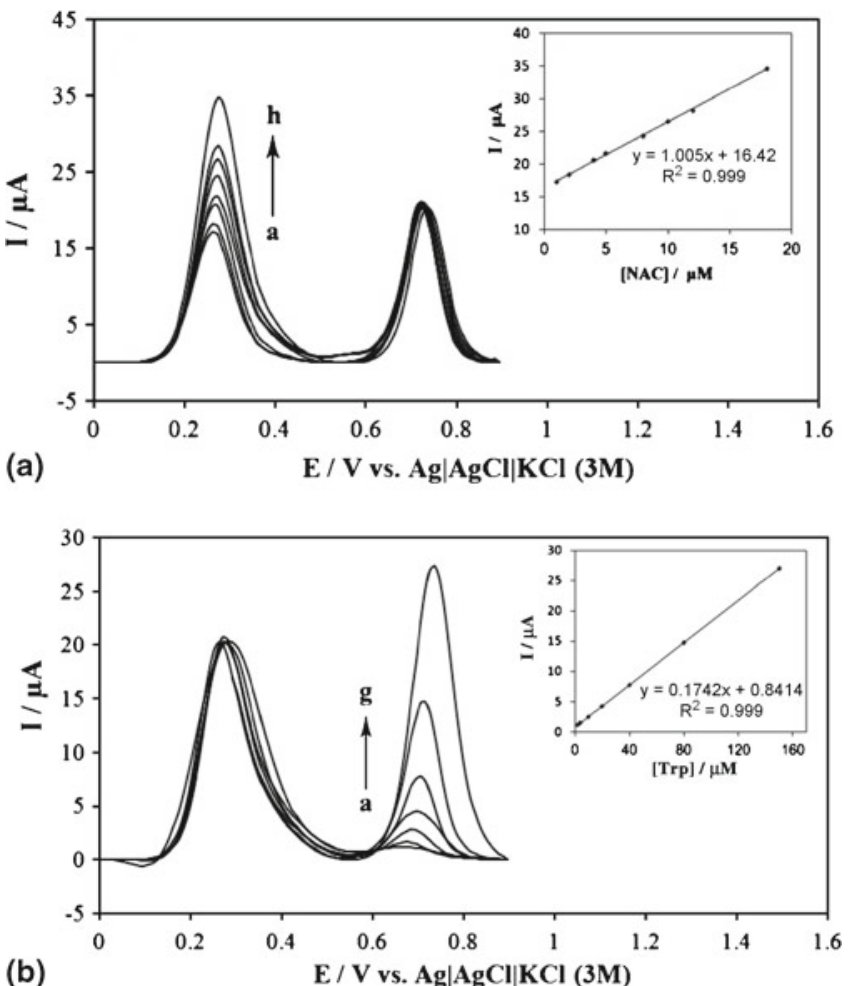

Figure 4. Differential pulse voltammograms of FCMCNPE in 0.1 M PBS (pH 7.00) containing (a) $100 \mu \mathrm{M}$ Trp and various concentrations of NAC: (a) 1, (b) 2, (c) 4, (d) 5, (e) 8, (f) 10, (g) 12 and (h) $18 \mu \mathrm{M}$; (b) $4 \mu \mathrm{M}$ NAC and various concentrations of Trp: (a) 2, (b) 4, (c) 10, (d) 20, (e) 40, (f) 80, (g) $150 \mu \mathrm{M}$. scan rate and pulse amplitude were $20 \mathrm{mVs}^{-1}$ and $50 \mathrm{mV}$, respectively. Insets $\mathbf{a}$ and $\mathbf{b}$ are calibration plots of anodic peak current vs. NAC and Trp concentrations, respectively. working electrode potential at $0.4 \mathrm{~V}$ (first potential step) and $0.2 \mathrm{~V}$ (second potential step) (not shown). As can be seen, there is no net cathodic current corresponding to the reduction of $\mathrm{FC}^{+}$to $\mathrm{FC}$ in the presence of NAC, when the second potential step is employed. While the forward and backward potential steps chronoamperometry on the modified electrode in supporting electrolyte shows very symmetrical chronoamperogram with an equal charge consumed for the oxidation and reduction of redox system into modified electrode matrix. The current corresponding to the electrochemical reaction of an electroactive compound (under diffusion controlled) is described by Cottrell's equation: ${ }^{40}$

$$
\mathrm{I}=\mathrm{nFAD}_{\mathrm{app}}^{1 / 2} \mathrm{C}_{\mathrm{o}} \pi^{-1 / 2} \mathrm{t}^{-1 / 2},
$$

where $\mathrm{D}_{\text {app }}$ and $\mathrm{C}_{\mathrm{o}}$ are the apparent diffusion coefficient $\left(\mathrm{cm}^{2} \mathrm{~s}^{-1}\right)$ and the bulk concentration $\left(\mathrm{mol} \mathrm{cm}{ }^{-3}\right)$, respectively. The plot of I versus $\mathrm{t}^{-1 / 2}$ represents linear dependency and from the slopes of experimental plots for different concentrations of NAC, the mean value of $\mathrm{D}_{\text {app }}$ was found to be $9.92 \times 10^{-6} \mathrm{~cm}^{2} \mathrm{~s}^{-1}$.

We have also used the chronoamperometric method to evaluate the heterogeneous catalytic rate constant according to the equation: ${ }^{41}$

$$
\mathrm{I}_{\mathrm{C}} / \mathrm{I}_{\mathrm{L}}=\pi^{1 / 2} \gamma^{1 / 2}=\pi^{1 / 2}\left(\mathrm{k}_{\mathrm{h}} \mathrm{C}_{\mathrm{b}} \mathrm{t}\right)^{1 / 2},
$$

where $I_{C}$ is the catalytic current of NAC at the modified electrode, $\mathrm{I}_{\mathrm{L}}$ is the limited current in the absence of NAC, $\mathrm{C}_{\mathrm{b}}$ is the bulk concentration of NAC, $\mathrm{k}_{\mathrm{h}}$ and $\mathrm{t}$ are the catalytic rate constant $\left(\mathrm{cm}^{3} \mathrm{~mol}^{-1} \mathrm{~s}^{-1}\right)$ and time

\begin{tabular}{|c|c|c|c|c|c|}
\hline Electrode & Modifier & Method & $\operatorname{LDR}^{\mathrm{a}}(\mu \mathrm{M})$ & $\mathrm{LOD}^{\mathrm{b}}(\mu \mathrm{M})$ & Reference \\
\hline $\mathrm{CPE}^{\mathrm{c}}$ & $\mathrm{CuHCF}$ & $\operatorname{LSV}^{\mathrm{d}}$ & $120-830$ & 0.63 & 15 \\
\hline $\mathrm{Pd}-\mathrm{Al}$ & Prussian blue & Amperometry & $2-40$ & 0.54 & 16 \\
\hline $\mathrm{GCE}^{\mathrm{e}}$ & Naphthoquinone / nanogold & DPV $^{\mathrm{f}}$ & $4.0-130$ & 0.8 & 17 \\
\hline GCE & Acetylferrocene & $\mathrm{CV}^{\mathrm{g}}$ & $600-6000$ & - & 39 \\
\hline CPE & $\mathrm{Fe}_{2} \mathrm{O}_{3} \mathrm{CoHCF}$ nanoparticles & LSV & $12.2-66.7$ & 0.205 & 42 \\
\hline CPE & $\mathrm{Fe}_{2} \mathrm{O}_{3} \mathrm{CoHCF}$ nanoparticles & Amperometry & $20-432$ & 0.0209 & 42 \\
\hline CNPE & cobalt(III) salophen complexe E & DPV & $0.1-100$ & 0.05 & 43 \\
\hline CNPE & $2,7-\mathrm{BF}^{\mathrm{h}}$ & DPV & $0.07-300$ & 0.052 & 44 \\
\hline CNPE & Ferrocene & DPV & $1.0-18.0$ & 0.49 & This work \\
\hline
\end{tabular}

Table 1. Analytical data obtained for NAC detection at the surface of different modified electrodes.

${ }^{a}$ LDR: linear dynamic range

${ }^{b}$ LOD: limit of detection

${ }^{\mathrm{c}} \mathrm{CPE}$ : carbon paste electrode

${ }^{\mathrm{d}} \mathrm{LSV}$ : linear sweep voltammetry

${ }^{\mathrm{e}} \mathrm{GCE}$ : glassy carbon electrode

${ }^{f}$ DPV: Differential pulse voltammetry

${ }^{\mathrm{C}} \mathrm{CV}$ : cyclic voltammetry

h2,7-BF: 2,7-bis(ferrocenyl ethyl)fluoren-9-one 


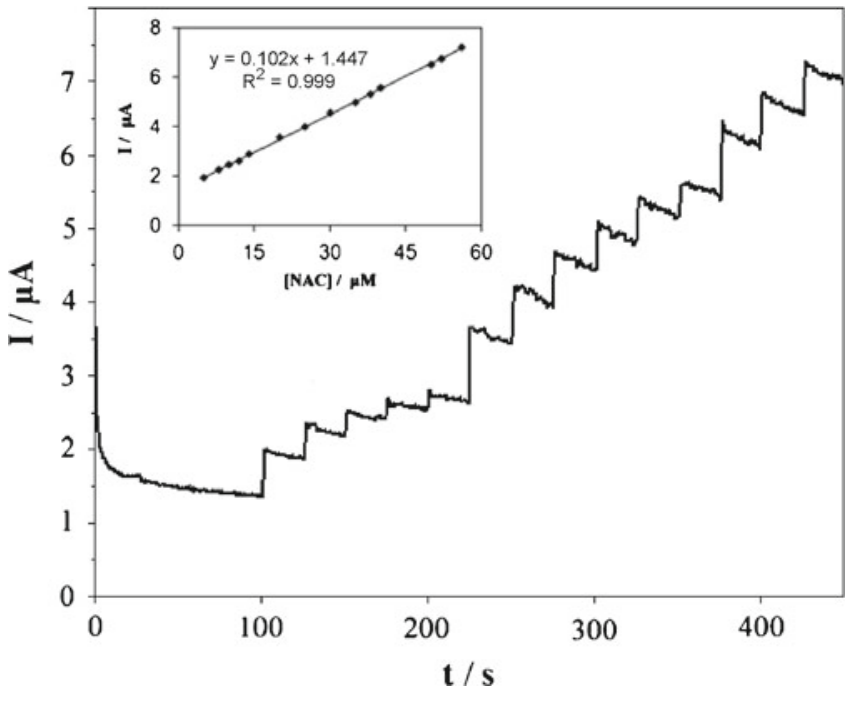

Figure 5. Hydrodynamic amperograms of FCMCNPE during the successive addition of NAC into PBS at constant electrode potential. Inset: The corresponding calibration plot for NAC.

elapsed (s). From the slope of $I_{C} / I_{L}$ versus $t^{1 / 2}$ plot, the value of $\mathrm{k}_{\mathrm{h}}$ was obtained as $3.07 \times 10^{5} \mathrm{~cm}^{3} \mathrm{~mol}^{-1} \mathrm{~s}^{-1}$ for a given concentration of NAC.

\subsection{Analytical measurement}

In order to evaluate the performance of the modified electrode, as a sensor for sensitive determination of NAC in the presence of Trp, analytical experiments were carried out by varying the concentration of NAC in the presence of fixed amount of $\operatorname{Trp}(100 \mu \mathrm{M})$ and varying Trp concentration in the presence of fixed amount of NAC $(4 \mu \mathrm{M})$ at $\mathrm{pH} 7.00$ at the surface of FCMCNPE using differential pulse voltammetry method. The DPV results show two well-distinguished anodic peaks at $270 \mathrm{mV}$ (NAC anodic peak potential) and $720 \mathrm{mV}$ (Trp anodic peak potential) potentials (figure $4 \mathrm{a}$ and $\mathrm{b}$ ). Thus, the response of the FCMCNPE to these two compounds was relatively independent. The calibration plots were linear within the concentration ranges of $1.0-18.0 \mu \mathrm{M}$ (with the correlation coefficient of 0.999 ) for NAC (inset in figure $4 \mathrm{a}$ ) and $2.0-150.0 \mu \mathrm{M}$ (with the correlation coefficient of 0.999 ) for Trp (inset in figure $4 b$ ). The obtained value of the detection limit $(3 \sigma)$ was to be $0.49 \mu \mathrm{M}$ and $0.54 \mu \mathrm{M}$ for the determination of NAC and Trp, respectively. As shown, we can use this method to measure Trp with high sensitivity too. The obtained analytical parameters for NAC determination at the surface of the modified electrode used in this research are comparable with the results reported by other research groups ${ }^{15-17,39,42-44}$ (table 1).

In order to develop another simple and sensitive sensing procedure for the analysis of NAC, hydrodynamic amperometry technique at constant electrode potential was also employed. Typical amperometric responses of FCMCNPE to successive addition of NAC and corresponding calibration graph are depicted in figure 5 and its inset, respectively. In the optimized conditions, the electrode response was linear within the concentration range of 5.0-56.0 $\mu \mathrm{M}$ for NAC and the detection limit $(3 \sigma)$ was $1.23 \mu \mathrm{M}$.

\subsection{Interference study}

In this work, the interference effects of some biologically compounds, such as $L$-cysteine ( $L$-cys), D-penicillamine (D-PA) and ascorbic acid (AA) on the electrode response to voltammetric determination of NAC were studied using DPV method. The results showed that $L$-cys and D-PA have interfering ability in voltammetric detection of NAC via increasing the sensor response. Although AA showed interfering effect on the electrode response to NAC in higher concentrations (about $1.0 \mathrm{mM}$ ), but in lower concentrations, it did not show interference in electroanalysis of NAC. Since AA content in red blood cells is not significant, ${ }^{45}$ it would have no influence on the electrode response to voltammetric determination of NAC in human blood serum samples.

\subsection{Real sample analysis}

In order to investigate the applicability of the proposed voltammetric method for the electrocatalytic determination of NAC in real samples, we have used the acetylcysteine tablets. The standard addition method was employed for measuring NAC concentration in the sample. The results of NAC concentration determination in this sample and recovery experiment obtained using

Table 2. The obtained results from NAC determination in pharmaceutical formulation $(n=3)$.

\begin{tabular}{lccccc}
\hline Sample & Amount added $(\mathrm{mM})$ & Amount found $(\mathrm{mM})$ & Recovery $(\%)$ & RSD $(\%)$ & $\mathrm{E}_{\mathrm{r}}(\%)$ \\
\hline Tablet & 0.200 & 0.1959 & 97.95 & 3.005 & -2.04 \\
\hline
\end{tabular}


this procedure are represented in table 2 , which demonstrate the accuracy of the proposed method and its efficiency for voltammetric determination of NAC in such samples.

\section{Conclusion}

In the present study, we have prepared a chemically modified electrode by incorporating FC in the matrix of CPE and CNPE. As it was found, FCMCNPE is compatible to FCMCPE because of the presence of CNT with many specific advantages such as large surface area and high conductivity; it can provide a suitable interface to promote the electron transfer reaction. The electrochemical behaviour of NAC in the presence of Trp at the surface of the prepared modified electrode was investigated by voltammetry. It is shown that The FCMCNPE is able to completely resolve the differential pulse voltammograms peak of NAC from Trp, revealing its good ability for simultaneous determination of these compounds in their mixture solution. The kinetic parameters such as transfer coefficient, catalytic rate constant, and diffusion coefficient for the oxidation of NAC were also determined. Also, the results of NAC concentration determination in tablet and the recovery experiment were obtained; demonstrate the accuracy of the proposed method and its efficiency for voltammetric determination of NAC in real samples.

\section{References}

1. Voet D and Voet J G 1995 Biochemistry, 2nd ed. (New York: John Wiley and Sons) p. 1263

2. De Viries N and De Flora S 1993 J. Cell Biochem. 53 270

3. Czap K 2000 Altern. Med. Rev. 5467

4. Liu M, Wikonkal M and Brash D E 1999 Carcinogenesis 201869

5. Valcour V and Shiramizu B 2004 Mitochondrion 4 119

6. Dekhuijzen P N R 2004 Eur. Respir. J. 23629

7. Zafarullah M, Li W Q, Silvester J and Ahmad M 2003 Cell. Mol. Life Sci. 606

8. Fu A L, Dong Z H and Sun M J 2006 Brain Res. 1109 201

9. Tsikas D, Sandmann J, Ikic M, Fauler J, Stichtenoth D O and Frolich J C 1998 J. Chromatogr. B: Biomed. Sci. Appl. 70855

10. Suarez W T, Vieira H J and Fatibello-Filho O 2005 Acta Chim. Slov. 52164

11. Al-Ghannam S, El-Brashy A and Al-Farhan B 2002 Farmaco $\mathbf{5 7} 625$

12. Chwatko G and Bald E 2000 Talanta 52509
13. Kochen W and Steinhart H 1994 L-TryptophanCurrent prospects in medicine and drug safety (Berlin: de Gruyter)

14. Nguyen N T, Wrona M Z and Dryhurst G 1986 J. Electroanal. Chem. 199101

15. Suarez W T, Marcolino L H and Fatibello-Filho O 2006 Microchem. J. 82163

16. Pournaghi-Azar M H and Ahour F 2008 J. Electroanal. Chem. 62222

17. Raoof J B, Ojani R, Chekin F, Jahanshahi M and RashidNadimi S 2009 Electroanalysis 212674

18. Raoof J B, Ojani R and Baghayeri M 2009 Sens. Actuators $B \mathbf{1 4 3} 261$

19. Shahrokhian S and Fotouhi L 2007 Sens. Actuators B 123942

20. Fouladgar M 2011 Int. J. Electrochem. Sci. 6705

21. Raoof J B, Ojani R, Beitollahi H and Hossienzadeh R 2006 Electroanalysis 181193

22. Ensafi A A, Khoddami E and Karimi-Maleh H 2011 Int. J. Electrochem. Sci. 62596

23. Merkoci A 2006 Microchim. Acta 152157

24. Gooding J J 2005 Electrochim. Acta 503049

25. Merkoci A 2007 Electroanalysis 19739

26. Wang J 2005 Electroanalysis 177

27. Zhang H, Meng Z, Wang Q and Zheng J 2011 Sens. Actuators B 15823

28. Tasviri M, Rafiee-Pour H A, Ghourchian $\mathrm{H}$ and Gholami M R 2011 J. Mol. Catal. B: Enzymatic 68206

29. Gopalan A I, Lee K P, Ragupathy D, Lee S H and Lee J W 2009 Biomaterials 305999

30. Raj C R and Chakraborty S 2006 Biosens. Bioelectron. 22700

31. Chakraborty S and Raj C R 2007 Electrochem. Commun. 91323

32. Gong K, Dong Y, Xiong S, Chen Y and Mao L 2004 Biosens. Bioelectron. 20253

33. Wildgoose G G, Banks C E, Leventis H C and Campton R G 2006 Microchim. Acta 152187

34. Jin G P, He J B, Rui Z B and Meng F S 2006 Electrochim. Acta $\mathbf{5 1} 4341$

35. Zhang M and Gorski W 2005 Anal. Chem. 773960

36. Musameh M, Wang J, Merkoci A and Lin Y 2002 Electrochem. Commun. 4743

37. Raoof J B, Ojani R and Chekin F 2009 J. Chem. Sci. 121 1083

38. Laviron E 1979 J. Electroanal. Chem. 10119

39. Gao Z N, Zhang J and Liu W Y 2005 J. Electroanal. Chem. $\mathbf{5 8 0} 9$

40. Bard A J and Faulkner L R 2001 Electrochemical methods: Fundamentals and applications (New York: John Wiley and Sons)

41. Galus Z 1976 Fundumentals of electrochemical analysis (New York: Ellis Horwood)

42. Heli H, Majdi S and Sattarahmady N 2010 Sens. Actuators $B \mathbf{1 4 5} 185$

43. Shahrokhian S, Kamalzadeh Z, Bezaatpour A and Boghaei D M 2008 Sens. Actuators B 133599

44. Beitollahi H, Raoof J B and Hosseinzadeh R 2011 Talanta 852128

45. Westerman M P, Zhang Y, McConnell J P, Chezick P A, Neelam R, Freels S, Feldman L S, Allen S, Baridi R, Feldman L E and Fung L W M 2000 Am. J. Hematol. 65174 